\title{
VICTUS ORDINE CONSTITUTO: LA ALIMENTACIÓN EN LA MEDICINA DE AMATO LUSITANO, UNA APROXIMACIÓN ${ }^{1}$
}

\author{
María Jesús Pérez Ibáñez \\ Universidad de Valladolid \\ Email: mariaje@fyl.uva.es \\ ORCID iD: https://orcid.org/0000-0002-9666-8413 \\ Victoria Recio Muñoz \\ Universidad de Valladolid \\ Email: victoria.recio @uva.es \\ ORCID iD: https://orcid.org/0000-0002-5661-2028
}

Recibido:11 marzo 2020; Aceptado: 21 junio 2020

Cómo citar este artículo/Citation: Pérez Ibáñez, María Jesús; Recio Muñoz, Victoria (2021) “Victus Ordine Constituto: la alimentación en la medicina de Amato Lusitano, una aproximación", Asclepio, 73 (1): p335. https://doi.org/10.3989/asclepio.2021/01

RESUMEN: Parte de la tarea médica del portugués João Rodrigues de Castelo Branco, pseudónimo de Amato Lusitano (1511-1568), consiste en atender o modificar la dieta de sus pacientes. A través de las prescripciones de la Centuria Tertia, uno de los libros que componen su obra Curationum Medicinalium Centuriae, podemos ver el amplio número de alimentos utilizados con fines terapéuticos, algunos realmente costosos. También conocemos cómo la atención de este médico por la dieta exige la obediencia del paciente.

PALABRAS CLAVE: Amato Lusitano; Curationum Medicinalium Centuriae; Dietética; Alimentación; Medicina del siglo XVI.

\section{VICTUS ORDINE CONSTITUTO: AN APPROACH TOWARDS DIET IN AMATUS LUSITANUS MEDICINE}

The medical practice of João Rodrigues de Castelo Branco, also known as Amatus Lusitanus (1511-1568), pays particular attention to the establishment or modification of the dietary customs of his patients. The prescriptions included in the third volume (Centuria Tertia) of his book Curationum Medicinalium Centuriae show the wide range of food products used for therapy purposes, some of them really expensive. It is also remarkable that Amatus demands obedience to his dietary regulations from his patients.

KEY WORDS: Amatus Lusitanus; Curationum Medicinalium Centuriae; Dietetics; Food; 16th Century Medicine. 


\section{INTRODUCCIÓN}

Desde Hipócrates (s. V a.C.) (De diaeta, De victus ordine, De alimento) y Galeno (s. II d.C.) (De sanitate

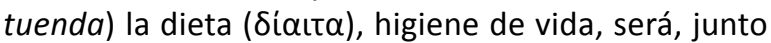
con la farmacia y la cirugía, uno de los pilares básicos en el tratamiento de la enfermedad y servirá de barrera contra agentes patógenos, pues a través de ella se puede llegar a mantener o recuperar el equilibrio de los humores, lo que entonces significaba tener una buena salud.

Según el esquema fisiológico diseñado por Galeno, la salud está determinada por la acción de una serie de factores externos, las denominadas sex res non naturales (aire y ambiente, comida y bebida, sueño y vigilia, lo que se expulsa y lo que se retiene, actividad y reposo y, por último, las pasiones del alma), sobre las que se puede intervenir, a diferencia de las res naturales, en las que no hay margen de maniobra (Álvarez de Palacio, 2008). Si desde Galeno la atención a la influencia de factores externos es evidente, es sobre todo en época bizantina y especialmente a partir de las traducciones de obras árabes en los siglos XI y XII cuando se establece una sistematización de estas sex res non naturales. A partir del siglo XIII esta concepción dietética se consolida en la obra de médicos como Taddeo Alderotti, Arnaldo de Villanova o Pedro Hispano y en un tipo de tratado denominado Regimen sanitatis dirigido bien a personas individuales (reyes o grandes señores) o referido a estados vitales (embarazo, esterilidad) y a la prevención de alguna enfermedad concreta como la peste (Albala, 2002, pp. 14-27; Nicoud, 2007; Cavallo; Storey, 2013, pp. 1-12).

En 1517 la versión latina de De sanitate tuenda de Galeno a cargo de Tomás Linacre (1460-1524) marcó un momento fundamental, pues se reactiva en cierto modo este interés por la dietética, impulsado también por la reedición del hipocrático De diaeta. Los historiadores consideran que la disponibilidad de estos tratados fundacionales y las nuevas posibilidades de difusión ofrecidas por la imprenta contribuyeron a que numerosos médicos escribieran tratados específicos de reglas de vida, basadas normalmente en la moderación y en una constante relación entre lo universal y lo particular. En este sentido, el buen clínico es capaz de adaptar las reglas generales a las exigencias de la complexión de su paciente, incluso a sus gustos (Andretta, 2011, pp. 287ss).

Amato Lusitano (1511-1568) ${ }^{2}$, pseudónimo del médico João Rodrigues de Castelo Branco, refleja este concepto de dietética en la práctica clínica que descri- be en su obra Curationum Medicinalium Centuriae ${ }^{3}$, siete libros publicados en diferentes momentos de su azarosa vida y que constituyeron un referente en el desarrollo del género médico de las Observationes (Pomata, 2010). Cada Centuria contiene cien curationes en las que el portugués describe casos clínicos que él mismo atendió acompañados, cuando lo juzga oportuno, de comentarios (scholia).

En sus casos observamos que una vía de curación consiste en aplicar los remedios terapéuticos oportunos (purgantes, sangrías, medicamentos, etc.) y atender las sex res non naturales. De los seis pares de elementos, la comida y la bebida ocupan un lugar destacado en la obra amatiana, consciente, como muchos de sus contemporáneos, de que una correcta alimentación influía enormemente en la salud del individuo.

Es cierto que el portugués regula también el ejercicio $(1.31 ; 3.1 ; 3.91)$, el sueño $(1.1 ; 1.40 ; 2.1)$, el aire $(1.31 ; 3.1 ; 3.43 ; 3.65)$, el baño $(2.77 ; 3.1 ; 3.91)$, las relaciones sexuales -abstención casi siempre- $(2.31$; $3.91 ; 6.55)$ o las emociones $(1.31 ; 3.43)$, pero con menos frecuencia. Las referencias a estos elementos aparecen sobre todo en aquellas curas en las que prescribe un régimen de vida completo y detallado, como en la 1.31, de cancroso quodam ulcere maligno et doloso mamillam infestante, el caso de una mujer recién parida a la que se le descubre una úlcera cancerosa en el pecho, donde va regulando para ella cada una de estas res non naturales:

Sic aggressus sum: primo sex res non naturales in ordinem disponendo ab aere inchoando. Aer igitur, sine quo nec sanitas conservari nec morbus pelli potest, ad temperiem semper declinabat, aestatis enim tempore ad frigus, hyemis vero ad caliditatem. Cibus porro talis describebatur qualis atra bile affectis convenit [...] Somnum potius longum et superfluum quam parcum esse permittebamus et illum quidem nocturnum [...] Exercitium quoque ante prandium temperatum permittebatur [...] Ab affectibus vero animae eam interdicere impossibile erat [...] Quantum vero ad evacuationem attinet, primo sanguinis extraxione (sic) usi sumus.

Así empecé a tratarla: en primer lugar, para regular las sex res non naturales empezamos con el aire. El aire sin el que no se puede conservar la salud ni expulsar la enfermedad tendía siempre a ser temperado, en verano más frío, en invierno más cálido. Se prescribía la comida que conviene a los enfermos de melancolía [...] Le permitíamos que el sueño fuera largo y abundante y no escaso y siempre nocturno [...] También se le permitía un ejercicio moderado antes de la comida [...] Era impo- 
sible que ella contuviera sus aflicciones [...] Por lo que respecta a la evacuación, en primera instancia, prescribimos una sangría.

Si las sex res non naturales son un factor que ha de tenerse en cuenta en la obra de Amato, nuestra atención se va a dirigir a aquello que concierne a la alimentación (a menudo asociada a la bebida) y vamos a referirnos, por acotar dentro de esta vasta obra, a lo que podemos leer en la Curationum Centuria Tertia, si bien presentamos ejemplos o referencias fuera de estos límites.

\section{LA ALIMENTACIÓN EN LA TERCERA CENTURIA DE AMATO LUSITANO}

Amato introduce de varias maneras la alimentación en sus procesos curativos, pues forma parte del diagnóstico y del procedimiento terapéutico, tal y como se atestigua desde antiguo.

Conocer la dieta previamente seguida por el paciente ofrece ayuda en el diagnóstico, como muestra en la reflexión (scholia) que acompaña al tratamiento de un niño de cinco años con viruela (curatio 3.16, de symptomate quodam ante variolarum eruptionem apparente): las evacuaciones previas a la erupción o la sangría posterior han de aplicarse en un momento de plethora, lo que determina entre otros factores a partir de la alimentación del niño (Sed id potissimum moliri debet quando plethora adest, quam medicus ex aegrotantis pueri habitu et anteacto victu ac symptomatis aegrum ipsum occupantibus exacte venari poterit).

Con todo, más que elemento diagnóstico, el alimento o su reglamentación actúa en el proceso terapéutico asociado o alternado con la medicación, como en 3.87, de coeliaco morbo et $\kappa \alpha \chi \varepsilon \xi i \alpha$ et $\alpha \dot{\tau} \rho \circ \varphi i \alpha$ :

De quo (sc. condito) singulis diebus singulas uncias ieiuno stomacho comedebat et desuper pro posse dormiebat et ad quatuor sequentes horas cibum non capiebat. lis enim et optimo victu, cuius inferius sermonem faciemus, intra viginti dies melius de ventriculo habere coepit.

De este preparado comía cada día dos onzas en ayunas y a continuación dormía todo lo posible y en las siguientes cuatro horas no tomaba alimento. Con esto y una excelente alimentación, sobre la que hablaremos después, en el plazo de veinte días empezó a sentirse mejor del estómago.

Más aún, alimentación y medicación aparecen como recursos equivalentes en 3.74, de febre maligna interficiente: lis addere possemus plures alias medicinas et varios cibos in sanguinem facile vertibiles nec minus varia et selecta composita.

A estas medicinas podríamos añadir muchas otras y varios alimentos que fácilmente se convierten en sangre y además varios selectos compuestos.

Este tratamiento puede llegar a ser único, si es el mejor posible. En 3.52, de febre acuta maligna, el alimento parece ser suficiente para revertir la fiebre del paciente:

Nihil medicaminum illi dabamus, solo victus ordine optime constituto contenti.

No le dábamos ningún medicamento, conformes con establecer un excelente régimen de alimentación.

En aquellos casos en los que hace referencia a la dieta sigue pautas que van de una simple mención a una precisa explicación:

a. Sus declaraciones más básicas sobre el victus "alimentación" o el victus ordo "régimen de alimentación" van acompañadas mayoritariamente por tres adjetivos: debitus "conveniente" (3.11; 3.33), bonus "bueno" (3.24; 3.67) y optimus "óptimo" (3.27; 3.56; $3.94 ; 3.96)$.

$3.11,[\ldots]$ cuius curas suscipiens ipse omnia, ut decet, cum debito victus ordine describo.

$3.11,[\ldots]$ al hacerme cargo de su cura, le prescribo, como se debe, todo lo necesario asociado a un conveniente régimen de alimentación.

3.24, Qui bono victus ordine gubernatus [...] hoc pharmaco liber evasit.

3.24, Este sometido a un buen régimen de alimentación [...] se curó con este fármaco.

$3.27,[. .$.$] optimo victus ordine constituto, quarto die$ in sudorem copiosissimum lapsa est et a febri libera evasit neque purgante pharmaco opus habuit.

$3.27,[. .$.$] tras mantener un óptimo régimen de alimen-$ tación, al cuarto día incurrió en abundante sudor y se libró de la fiebre y no necesitó ningún medicamento purgante.

b. Esta actuación sobre la alimentación del paciente puede llevarle a aplicar formas específicas de dieta, aunque esto no significa que las detalle. Entendemos que son formas o tipos conocidos o comunes para los médicos lectores de su obra.

Por ejemplo, las dietas recomendadas pueden ser tenues $(3.53 ; 3.87 ; 3.92)^{4}$ o bien subtiles como en el caso de un varón de 35 años que sufre dolor de cabe- 
za como consecuencia de la herida en un ojo (3.7, de ingenti capitis quodam dolore post oculum confossum superveniente). Amato recurre a un procedimiento curativo mediante sangrías y parece completar el tratamiento con una modificación de la dieta en un sentido muy concreto:

Topica vero medicamenta parum iuverunt, sed primo [...] aeger bolum ex cassia deglutivit et victus ratio subtilis admodum fuit.

Los medicamentos tópicos le aliviaron ligeramente, si bien al principio el paciente tomó un bolo de casia y su régimen de alimentación fue esencialmente ligero.

De forma similar actúa en uno de los varios casos de viruela (3.18, de variolis serpentibus, cutem tantum erodentibus) en el que una forma concreta de alimentación es el complemento necesario a la acción de medicamentos refrigerantes aplicados sobre el hígado: victus ad id multum faciebat, qui ad frigidum cum humiditate declinabat. Además, en 3.43, de cordis palpitatione, para una religiosa de 20 años, que empieza a tratar con sangrías y siropes, prescribe una dieta con cualidades secantes y atenuantes, Victus ratio in universum exiccatoria et attenuatoria erat, sed optimi succi ${ }^{5}$.

Estas dietas específicas, pero no detalladas, también se relacionan con un humor o cualidad como en 3.55 , de quodam musico varias imaginationes et phantasmata diversa habente, para cuyo paciente prescribe una dieta similar a los afectados por melancholia /atra bilis:

Caeterum huic victus, qualis atrabile affectis debetur, concedebatur, non minus quod inter amicos semper versaretur et solitudines fugeret eum monebamus.

Además, le recomendábamos la alimentación que debe prescribirse a los enfermos de melancolía, y mucho más le aconsejábamos rodearse siempre de amigos y evitar la soledad.

O en 3.56, de iuvene Hebraeo puellae Hebraeae amore capto, el caso de un joven judío enamorado de una joven judía:

Huius igitur nos curam suscipientes, optimo victus ordine, pro ut atra bile affectis convenit constituto, illi ex elleboro syrupum compositum [...] dabamus.

Al asumir nosotros su curación, tras prescribirle un régimen de alimentación óptimo, como conviene a los enfermos de melancolía, le dimos un preparado de sirope de eléboro.

Puede ser un poco más preciso, en tanto que establece "categorías de alimentación", como en 3.95, de febre nocturna miti et lenta simulque de hemitritaeo, id est semitertiana febre, con un febricitante que incurre en semiterciana y cuyo estómago le preocupa. En los scholia, casi un monográfico sobre este tipo de fiebre, apunta los modos de alimentación que considera necesarios para estos pacientes con un habitual en él, pero poco clásico scire decet quod:

Sed circa ordinem tradendi victum iis qui semitertiana laborant scire decet quod eo die, quo uno tantum aeger affligitur paroxysmo, copiosius et liberalius cibandus est; sequenti vero die parcius et subtilius tractandus est, quia natura duabus febribus attenta, afflicta et gravata comperitur.

En relación con el orden en que deben tomar alimento quienes padecen semiterciana conviene saber que en el día en el que el enfermo padece un solo paroxismo, puede ser alimentado con mayor abundancia y generosidad; al día siguiente ha de tratársele de forma más parca y sutil porque la naturaleza alcanzada por las dos fiebres se encuentra afligida y agravada.

c. Si la forma de dieta requerida, por conocida o común, puede expresarse de esta forma que podríamos calificar de elíptica, también puede ser precisada con creciente nivel de detalle.

En la curatio 3.65, de gurgulione plus iusto longo facto simulque de raucedine, refiere el caso de un paciente con la úvula elongada asociada a una ronquera, al que casi ni se le oye cuando habla y apenas puede comer. Entiende que para curarlo debe estudiar su temperamento y percibir que está acostumbrado a mucha comida, mucha bebida y mucho sexo (multo victui et potui assuetus et Veneri summopere deditus). Todo esto le lleva a sospechar que su enfermedad es una secuela del morbo gálico (Pérez Ibáñez, 2019). En este punto inicia Amato lo que podríamos denominar una digresión explicativa que podemos asociar con la voluntad didáctica que en tantas ocasiones deja ver, pues aclara que las referencias a la terapia aplicada, dieta incluida, se hacen para que las conozcan quienes practican la medicina, pues es un tipo de intervención común en enfermedades de la misma tipología:

Referam tamen ipse (ut medicam artem exercentes noscant) ordinem a nobis primo habitum et primo a victus ordine initium sumam, quo vel tantum similes morbi sanari consuescunt.

Por mi parte yo mismo voy a exponer (para que lo conozcan quienes ejercen la medicina) el orden que hemos mantenido desde el principio y empezaré por el régimen de alimentación, solo con el que acostumbran a sanarse enfermedades similares. 
Con todo, sus especificaciones son todavía bastante genéricas, por así decir, pues se limita a señalar tipos de alimentos que el paciente debe evitar, sin entrar en menciones particulares. Todos ellos comparten la condición de ser alimentos pituitosos (cibaria fugiant pituitosa, qualia sunt pisces, suillae carnes, lacticinia, legumina, immoderata iuscula, herbae et caetera) de la misma forma que propone que debe tomar aquellos alimentos que generan buenos jugos (optimum succum gignentibus cibis) ${ }^{6}$.

Y continúa su prescripción con recomendaciones generales que ese buen práctico ha de tener en cuenta (unum cordi praefixum habens quod-de nuevo con ese poco clásico estilema que suele ser recurrente en él-): el almuerzo ha de ser más copioso que la cena e incluso, si la comida es muy abundante, puede prescindirse de la cena (prandium coena maius ac liberalius semper accipiebat, imo cum prandium opiparum illi erat, a coena nostris monitis abstinebat) ${ }^{7}$. Esta reflexión se amplía también al vino, tanto en la comida como en la cena ${ }^{8} y$ el sueño, pues en este caso el diurno debe ser evitado:

Vinum, [...] in prandio tantum bibebat et illud quidem parvum, caput non feriens. In coena autem id omittebat, quoniam eius halitu caput repleri et cerebrum tentari aiebat.

Vino, [...] bebía solo en el almuerzo y poca cantidad, para no dañar la cabeza. En la cena, en cambio, lo obviaba, pues decía que la cabeza se llenaba de sus vapores y afectaba al cerebro.

Somnum quoque diurnum, utpote caput replentem et noxas plures secum ferentem, ut poterat, fugiebat, veluti illico a coena

Evitaba, lo que podía, el sueño diurno ${ }^{9}$, porque aturde la cabeza y provoca muchos perjuicios, como también después de cenar.

d. Amato puede dar indicaciones muy concretas sobre qué alimentos deben ingerir sus pacientes. Por ejemplo, en uno de los varios casos de viruela (3.15, de variolis et morbillis, exanthematis dictis, de eorumque symptomatibus ante apparitionem venientibus) para su paciente de seis años prescribe alimentos muy precisos según las distintas fases de la enfermedad y diferencia entre los de la fase previa a la aparición de las pústulas y los requeridos post exactam earum eruptionem, sobre todo carne de pollo o capón.

e. En un nivel máximo de precisión encontramos varias curaciones $(3.1 ; 3.11 ; 3.66 ; 3.86 ; 3.87$ y 3.91$)$ que comparten el rasgo de ofrecer una detallada relación de alimentos, incluso de preparaciones viables. Vistas en su conjunto las curationes de la Tercera Cen- turia ofrecen una abundante cantidad de productos posibles, algunos bastante recurrentes.

Quizá no sea una coincidencia que algunos de estos casos para los que es minucioso detallando la alimentación vengan relacionados con un paciente desobediente a los preceptos dietéticos del médico (3.11, de quodam praeceptis medicis non obediente), con enfermedades intestinales (3.66, de acri humore a capite ad os descendente et aphtas inibi gignente et postea dysente-

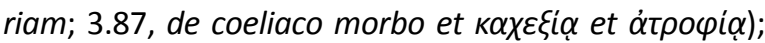
trastornos en la alimentación (3.86, de citta, id est pica morbo, quem $\mu \alpha \lambda \alpha \kappa i \alpha v$ quoque quasi mollitiem medici appellant) o fiebres consuntivas (3.1, de hectica simulque de marcore sive tabe) -en las que el exceso de ingesta se considera nocivo ${ }^{10}$-. En la cura 3.91, de puero absque manifesta causa faciem tumentem habente, afirma que, si no le cambiara la dieta, la afección que ha detectado en el hígado evolucionaría a hidropesía. Aquí presta mucha atención a la alimentación de este paciente, aunque resulta interesante observar cómo en esta misma cura a otro niño, casi seguramente de familia menos pudiente (ruri autem hic agebat), le prescribe una dieta mucho menos variada y refinada: una simple papilla de trigo (pulticula) apenas aderezada con poleo y orégano acompañada de vino como purgante.

\section{LA “DESPENSA” DE AMATO LUSITANO}

Gracias a estas curaciones tenemos una amplia visión de la posible "despensa" médica que propone Amato, aunque no sea esta la despensa completa. No podemos olvidar que muchos de estos elementos pueden aparecer como alimentos o integrarse en las preparaciones medicamentosas que el propio Amato inserta en su relato como ocurre en las ya mencionadas 3.66 y 3.91 .

Siguiendo las especificaciones de nuestro médico en esa despensa encontramos:

1. Diversas carnes, entre ellas la de ovicápridos: cabrito $(3.1 ; 3.86 ; 3.87$; 3.91), carnero $(3.86 ; 3.87 ; 3.91)$; bovinas (iuvencula bovis lactans 3.1) y también tienen cabida liebres $(3.87 ; 3.91)$ y conejos pequeños $(3.87$; 3.91), animales impuros según las leyes judías. Sin duda, la carne más mencionada es la de aves, salvajes y domésticas (volucres montanae et domesticae): capones (3.1; 3.86; 3.87; 3.91), pollos de gallina o gallina (3.1; $3.86 ; 3.87 ; 3.91)$, pollos de paloma doméstica (3.87), palomas (3.87), papamoscas (3.87), tórtolas $(3.87 ; 3.91)$, faisanes $(3.1 ; 3.86 ; 3.87 ; 3.91)$, perdices $(3.1 ; 3.86$; 3.87; $3.91)$, tordos $(3.86 ; 3.91)$, mirlos $(3.86 ; 3.91)$, attagenae 
o francolines ${ }^{11}(3.1 ; 3.87 ; 3.91)$, considerados "boccato di cardinale" (1.31: Romae Cardinalium cibum appellant), las gallinas africanas o pintadas, una exquisitez en Roma $^{12}$ (3.87; 3.91). Se prefieren, además, jóvenes en la idea de que se digieren mejor y mantienen el equilibrio humoral (Banegas López, 2012, pp. 60-61).

Apenas encontramos carne de cerdo. Hemos visto (3.65) que prefiere evitarla por excesivamente pituitosa. En otras ocasiones la prohíbe expresamente y a veces apoyándose en Hipócrates (Hp. epid. 7,82 [L. $5.436-438]^{13}$ ) limita su prohibición a los meses de verano (2.32).

La carne puede prepararse de diversas maneras, asada o hervida:

3.91, Quas (sc. carnes) potius assas quam aqua coctas comederet suadebam. Si quando tamen elixae parabantur, herbis iis condiebantur: hyssopo, mentha, satureia, maiorana, cerepholio, foeniculo, basilicone, porro vulgari petroselino et similibus adiectis aromatis croco et gariophylis, cinnamomo et caro.

3.91, Aconsejaba comer carnes asadas mejor que cocinadas en agua. Si alguna vez las preparaba hervidas, las condimentaba con estas hierbas: hisopo, menta, ajedrea, mejorana, perifollo, hinojo, albahaca, además perejil común y otras aromáticas semejantes como azafrán, clavo, canela y alcaravea.

Y de forma más habitual como contusum o pistum $(1.21 ; 3.87)$ la sustancia que se obtiene de la carne de ave, especialmente de la gallina o perdiz, tras machacarla y prensarla (Herrera Hernández, 1996, col. 1230) acompañada de aderezos ${ }^{14} \mathrm{y}$ de ptisana o cremor ptisanae ${ }^{15}$ :

3.1, contusum ex carnibus caponum, pullorum gallinaceorum, haedi et nonnunquam iuvenculae bovis lactantis, non nisi frigidis seminibus apparatum, cui saepe cremoris ptisanae tertiam partem misceri mandabamus.

3.1, puré de carnes de capón, pollo, cabrito y a veces de ternera de leche, también aderezado con semillas frías al que a menudo solíamos mandar añadir una parte del residuo de la tisana colada.

3.71, pulli contusum ptisanae mixtum.

3.71, puré de pollo mezclado con tisana.

3.87, Ex quorum carnibus succus eo modo a nobis paratus concinnabatur veluti contusum pistum dicunt.

3.87, Con el jugo de esas carnes que preparamos de ese modo se elaboraba algo así como un puré que llaman pisto.
Y en forma de caldo:

3.71, capi ius in quo semina citri, acetosae, bolus Armeniae, margaritae praeparatae, corallus praeparatus et iis similia in pulverem redacta mittebantur.

3.71, caldo de capón en el que se echaban semillas de cidra, vinagrera, bol arménico, perlas y corales preparados y otros semejantes previamente pulverizados.

Las indicaciones sobre el caldo de capón, gallina o perdiz, que considera el mejor porque se digiere fácilmente, no daña el vientre y apenas genera humores superfluos, alcanzan la independencia de una receta de cocina.

3.87, (sc. succum capi aut gallinae vel perdicis) sic extrahere mandabamus: alitem exenteratam accipe et ipsam optimo et puro mastice cum cinnamomo selecto, quod facile in officinis et tabernis habetur, et pauculo pane refarci et ad ignem in veru coque. Quam sic non exacte assam torculari exprimes et succum mulctum inde (ut ab amygdalis oleum extrahitur) aegro potandum dabis, non nisi pulvisculo specierum diamoschi et diambrae adiecto.

3.87, (sc. jugo de capón, de gallina o de perdiz) así mandábamos extraerlo: se toma un ave eviscerada y se rellena con excelente y puro mástique junto con canela selecta, que fácilmente se encuentra en establecimientos y tiendas, y con un poco de pan; se pone al fuego en una espeta. No asada del todo se aprieta en una prensa y el abundante jugo que sale (como se extrae el aceite de almendras) se da a beber al enfermo acompañado de polvo de las especias del diamosco y del diámbar.

Otra preparación común de la época en la que puede haber carne es la panatella (panetela) ${ }^{16}$, una forma de gachas a base de diversos caldos, sobre cuya preparación en ocasiones da instrucciones muy precisas:

3.66, constituimus ut panatellam ex farinae flore, quem similaginem vocamus, concinnatam pauculo butyro adiecto comederet vel similaginis loco orizae pulverem poneret aut ex iure pedum vituli aut nephrendis vervecisve praedicta panatella pararetur commendabamus; cui aromatum vice pulverem ex bolo Armeniae, thure, mastiche utroque corallio et similibus inspergebant.

3.66, establecimos que comiera panetela preparada con la flor de harina, que llamamos sémola, a la que se le añade un poco de mantequilla o que en lugar de la sémola pusiera arroz pulverizado o le recomendábamos que preparara esa panetela con caldo de pata de ternera, de cerdo o de carnero castrados; se sazonaba a su vez con polvo aromático de bol arménico, incienso, mástique, coral y similares. 
2. Igualmente son frecuentes los huevos, a ser posible recientes (3.86), de los que se pueden utilizar solo la yema y la clara $(3.1 ; 3.66)$ o bien la yema mezclada con agua de rosas (3.71).

3. En cuanto a los pescados tienen cabida tanto los marinos como los de agua dulce (tam marini quam dulci aqua natantes) (3.1) y de forma más específica menciona los petrosi "de roca" $(3.1 ; 3.86)$.

4. También se incluyen todo tipo de especias y hierbas: semillas frías (semina frigida, 3.1), cilantro verde o seco (3.1), azafrán (3.1; 3.87; 3.91), mástique (3.87), canela (3.87; 3.91), nuez moscada (3.87), pimienta (piper longum, 3.87), clavo (3.87; 3.91), poleo (3.91), orégano (3.91), menta y eneldo (3.86), hisopo (3.87), perejil $(3.87 ; 3.91)$ y condimentos como el agraz (omphacium, 3.1)

5. En sus dietas aparecen todo tipo de verduras y hortalizas (olus): calabaza (3.1); espinacas, armuelle blanco, endivia y verdolaga (3.86), alcachofa (3.87) y perifollo, que considera desconocido en Hispania (3.87: cerepholio, herbula universae Hispaniae (ut puto) ignota). Desaconseja las legumbres (3.87) y recomienda el arroz (3.87).

6. Se hallan frutos tanto frescos (horarii): uvas, higos, melones, granadas, cerezas, ciruelas (3.1), olivas en salmuera (3.87), almendras (3.87), uvas pasas (3.87); como secos (ex sole siccatis): ciruelas, cerezas, almendras, pistachos y piñones, estos últimos principalmente azucarados (3.1).

7. Un grupo muy amplio es el de los alimentos dulces y golosinas (tragemata et bellaria) que suelen cerrar su exposición de productos recomendados.

3.1, martii panes, semen melonis saccharo incrustatum, amygdalae saccharo coopertae, et huius generis saccharo confecta bellaria et tragemata.

3.1, mazapanes, semillas de melón rebozadas en azúcar, almendras garrapiñadas y otros dulces y golosinas de este tipo elaboradas con azúcar.

3.86, martii panes, pinosaccharatum, uva passa exiccata et similia.

3.86, mazapanes, piñones azucarados, uvas pasas secas y otros semejantes.

3.91, uva passa, pistacia, quae vino malvatico macerata summam praerogativam in hoc habent casu, amigdalae et pineae, quibus addas velim, cappares ex oxymelite paratos, asparagos et foeniculum marinum, cretamum dictum.
3.91, uvas pasas, pistachos, que macerados en vino de malvas son de mucha ayuda en este caso, almendras, piñones, a los que añade, por favor, alcaparras preparadas con ojimiel, espárragos e hinojo marino llamado cretamum.

En ocasiones se detiene en dónde y cómo se confeccionan:

3.87, Pino saccharatum multum laudatur et Martii panes, veluti condita altera palmaris longitudinis, suavia, cinnamomi colorem referentia, quae Venetiis, a moscho forte quo ornantur, moscacholia vel mostacholia vocantur, et eorum vice Lusitani Hispani canellones suavissimos et cordiales admodum parant, ut varia alia condita saccharaea, moscata et ambarata praeteream.

3.87, Muy de alabar son los piñones azucarados y los mazapanes, así como otros preparados de la longitud de una palma, que recuerdan el color de la canela y que en Venecia, quizá por el almizcle con el que los adornan, llaman moscacholia o mostacholia ${ }^{17}$ y por su parte los Lusitanos de Hispania preparan unos canellones muy dulces y agradables, por no referirnos a otros preparados azucarados, moscados, almizclados y ambarinos.

La bebida no siempre es expresamente mencionada y en estas curaciones hemos encontrado dos: vinum (González Manjarrés, 2007) y aqua hordei "agua de cebada" u hordeacea (3.1), "hordiate" en castellano ${ }^{18}$.

Parte de estos alimentos, calificados como selectos y mejores, son los que recomienda en el caso de la paciente afectada del trastorno alimentario llamado "pica", en virtud de la cual tomaba absurdissimos cibos.

3.86, Curaturi igitur nos puellam hanc primo quod optimis et selectis uteretur cibis proposuimus, veluti carnibus phasianorum, perdicum, turdorum, merularum, caponum, gallinarum, haedi, veruecis et similium. Non minus utebatur ovis recentibus, piscibus petrosis et aliis optimi succi cibariis. Ex tragematis et bellariis accipiebantur martii panes, pinosaccharatum, uva passa exiccata et similia. Ex oleribus vero permittebamus spinachiam, aureum olus, atriplicem dicimus, albam endiviam, portulacam, mentham et anethum.

3.86, Al ir a tratar a esta muchacha primeramente le propusimos que tomara excelentes y selectos alimentos como carne de faisán, perdiz, tordo, mirlo, capón, gallina, cabrito, carnero castrado o similares. También tomaba huevos recientes, peces de roca y otros alimentos de buen jugo. De entre los dulces y las golosinas se tomaban mazapanes, piñones azucarados, uvas pasas 
secas y semejantes. De las verduras le permitíamos la espinaca, el armuelle, decimos el armuelle bueno, la endivia blanca, la verdolaga, la menta y el eneldo.

\section{AMATO Y LA ALIMENTACIÓN DE SUS PACIENTES, OBEDIENCIA DEBIDA}

Más allá de la amplitud, detalle o variedad de la que hemos denominado "despensa" de Amato, llama la atención la importancia que concede a la obediencia por parte del paciente de la pauta dietética que se le ha prescrito. Así ocurre en la curatio 3.11, expresivamente titulada de quodam praeceptis medicis non obediente.

Por medio de un diálogo fingido Amato incide en otro tema muy de su gusto: la relación médico-paciente, la confianza que genera en el enfermo la solvencia del sanador, temas que aborda en el Introitus que a modo de introducción encabeza sus Curationum Centuriae (Recio Muñoz, 2018). En ella podemos ver que no es tan importante el proceso nosológico concreto como la actitud del paciente. El protagonista de esta curatio es un noble romano de la familia de los Colonna que puede identificarse con Camillo Colonna (XV'$1558)^{19}$, al que trata durante su estancia en Roma ${ }^{20}$.

Amato nada dice de la complexión, antecedentes o enfermedad que sufre el paciente ${ }^{21}$. Al asumir su tratamiento corrige su alimentación (cuius curas suscipiens ipse omnia, ut decet, cum debito victus ordine describo), al parecer con poco éxito, pues el enfermo no le hace caso e incurre en nuevos y graves síntomas, algo que le desveló un ayuda de cámara (unus cubicularius, et qui victum porrigit, rem ex toto aperuit). En este punto introduce Amato una primera reflexión sobre la conveniencia de interrogar al servicio como fuente segura de información basándose en el ejemplo de Galeno ${ }^{22}$ De praecognitione ${ }^{23}$ (= De praenotione ad Epigenem [K. 14.599-673]).

Decet autem medicum priusquam ad aegrotum veniat, domesticos interrogare et de eius statu ac dispositione percontari, quo facto raro vel nunquam aberrabit. Hac enim via incedens Galenus multa praeterita aegrotantibus obiiciebat, quae tanquam miracula ab eis habebantur, ac postea maiori cum animo sese illi concredebant, ut ex libro De praecognitione facile quis iudicare poterit.

Es bueno que el médico antes de acercarse al enfermo interrogue a los de la casa y les pregunte sobre el estado y disposición del paciente, con lo que rara vez o nunca se equivocará. Siguiendo este camino Galeno echaba en cara a sus pacientes muchas cosas pasadas lo que ellos tenían por milagroso y luego con mayor ánimo se confiaban a él, como a partir del libro De praecognitione se puede deducir.

Ciertamente se trata de una cita ideológica, no literal, casi un resumen (muy orientado en su beneficio) de los mecanismos de diagnóstico mencionados por Galeno en el capítulo sexto de su obra; una obra que, por lo demás, está concebida como un diálogo continuo entre el autor (Galeno) y su interlocutor (Epígenes) y en ocasiones la narración se anima con explícitas referencias de Galeno al tú de su interlocutor y a menciones de lo que este interlocutor dice o pregunta (ait, inquit o inquirit). Forma esta de actuar que, a nuestro entender, podría explicar que Amato inserte a continuación un diálogo con su paciente que retransmite tanto por la vía de la subordinación sintáctica (estilo indirecto), como por la presuntamente literal reproducción de las palabras (estilo directo).

Convenientemente informado y simulando no saber nada, Amato interroga a su paciente sobre las causas de ese malestar sobrevenido (ego tanquam rei inscius simulo, alioqui quaeritans unde haec evenire poterant) quien responde que lo ignora. Amato le asegura que se ha excedido en la alimentación (ego vero in victu ipsum peccasse contestabar) y se nos va desvelando la verdad de una cena que el paciente no considera basada en alimentos nocivos:

Cum rem ex toto tenerem, quidnam in coena nocte praeterita accepisset inquiro. Ille nihil noxii aut mali succi respondet; tunc hominem rogo quid illud sit singulatim recenseat.

Cuando ya lo tenía del todo claro, le pregunto qué ha ingerido la pasada noche en la cena. Él responde que nada dañino o de malos jugos; entonces le ruego que me los detalle uno por uno.

En este punto, pasando a una transcripción literal de la conversación, conocemos en un relato en primera persona el menú de la cena de un noble romano:

Ille "primo capi carnes, a quibus omnino abhorreo, gustare coepi" -ait-, "dein frixa ova mihi apposita sunt, quae tantillum gustavi"; demum "ex ostracodermis unum vel aliud genus lubetissime comedi", asserit.

Y él "al principio empecé a tomar carne de capón" -dice- "que no me gusta nada. Luego huevos fritos, que apenas probé"; y finalmente asegura "comí con mucho gusto una o dos clases de marisco".

Ante tamaña desobediencia que Amato le reprocha, se retira de su cuidado, algo que se ocupa de 
trasmitir al Pontífice, los Cardenales y otros príncipes romanos.

Tunc ego submissa voce, "haec promissis respondere", aio, ac venia demandata recessi, non rediturus ad illum tametsi unum vel alterum ex suis castris mihi dono daret. Novit haec summus Pontifex novereque plures reverendissimi Cardinales ac alii principes Romani.

Entonces yo en voz baja me digo "Así responde a sus promesas" y pidiendo la venia me retiré y no he de volver a visitarlo, aunque me regalara uno o dos de sus castillos. Saben esto el Sumo Pontífice y muchos reverendísimos Cardenales y otros príncipes romanos.

Esta actuación de Amato responde a los postulados éticos con los que abre las Curationum Centuriae, como hemos señalado, y viene avalada por la inserción de unos scholia que declaran que el paciente debe obedecer a su médico. Esta idea la refuerza con la declaración de que tiene más valor para él la verdadera medicina que el dinero ${ }^{24}$.

Languens medico obedire debet, ut mandat Hippocrates primo Aphorismorum (Hp. Aph. 1,1 [L. 4,458]) et libro primo De morbis vulgaribus (Hp. Epid. 1,5 [L. 2,636] cf. Gal. Hipp. Epid. Comm. 2,50 [K.17.1,150]), unde iis qui medicorum praeceptis non obsequuntur, medicinale consilium dandum non est, ut suadet Galenus libro De sanguinis missione (Gal. Cur. Rat. Ven. Sect. 7 [K. $11,272]$ ) et sexto De tuenda valetudine (Gal. San. Tu. 6,8 [K. 6,415]) et primo De diebus decretoriis (Gal. Di. Dec. 1,11 [K. 9,825]). Nec enim apud me pecuniae, quas illustrissimus vir hic mihi liberaliter elargiebatur tantum unquam valuere ut eas artis medicae nobilitati et excellentiae anteponerem, sciens utique quod sapientia propter seipsam expetenda est, non vero propter lucrum, ut suadet Galenus libro illo cui titulus est Quod optimus medicus sit et philosophus (Gal. Opt. Med. [K. $1,57 ; 1,61]$ ) et secundo Methodi medendi (Gal. MM 2,5 [K. 10,114-115]), veluti sexcentis aliis locis.

El paciente debe obedecer a su médico, como manda Hipócrates en el primero de los Aforismos y en el libro primero de las Epidemias, de donde se desprende que a quienes no siguen las prescripciones de su médico, no hay que darles consejos médicos, como también nos sugiere Galeno en el libro de La sangría, en el sexto de La conservación de la salud y en el libro primero de Los días críticos. Tampoco para mí tenían tanto valor las riquezas que ese muy ilustre señor me prodigaba generosamente, que llegara a anteponerlas a la nobleza y dignidad de la profesión médica, sabiendo por lo demás que la sabiduría ha de buscarse por sí misma y no por afán de lucro, tal como Galeno aboga en el libro titula- do Que el mejor médico también sea filósofo, en el libro segundo del Método terapéutico y en otros mil pasajes.

Toda esta declaración se apoya en la común batería de autoridades que le han servido para sustentar esos mismos postulados en el Introitus ${ }^{25}$. Si miramos con detalle cómo en estos scholia introduce las citas podemos ver que son referencias, en general, ideológicas y moduladas en función de sus intereses. A modo de ejemplo en la cita De sanguinis missione el texto que encontramos en los Opera omnia de 1544 (Galeno, 1541-1545, 5.95) dice Eis vero qui praeceptis salutaribus obsequentur, summopere profueris, donde apoya claramente la dedicación del médico en relación con el paciente que sigue sus indicaciones, pero no se afirma lo contrario, como hace Amato.

Si la alimentación no tuviera tanta importancia en el tratamiento que Amato prescribe a sus pacientes, este caso clínico parecería extemporáneo. Como alimentación y tratamiento son, como podemos ver, dos caras de la misma moneda no es difícil comprender el enfado de Amato ante la violación de sus prescripciones y que un caso como este, que aparentemente no tiene interés médico, forme parte con entidad propia de las Curationum Centuriae y además presente un comentario.

A pesar de la rigidez con la que trata a este paciente desobediente, Amato se muestra más flexible y tolerante en otras ocasiones. Así tiene en consideración los gustos alimenticios de los pacientes, como el caso de un enfermo que odiaba por naturaleza el dulce y al que le prescribe solamente alimentos salados, ácidos y agraces (6.60, de quodam qui natura sua multa et varia abhorrebat). 0 bien respeta las restricciones alimentarias asociadas a la religión. En la curación 2.18, de quodam qui rei Venerae uti non poterat, aborda la cuestión de los afrodisiacos que ha de tomar un paciente que sufre de impotencia y, en concreto, sobre el pescado explica que le recomienda aquellos permitidos por la ley (mosaica) ${ }^{26}$ :

Sed et pisces in lege admissos concedimus [...] quanquam multi corio tecti veluti testa cooperti libidinem incitent, sed quia huic per religionem vetiti sunt, eos silentio praterire satius est.

$Y$ le permitimos el pescado admitido en su ley [...] aunque muchos crustáceos recubiertos de caparazón excitan la libido, pero como le están prohibidos por su religión, es preferible no mencionarlos.

\section{CONCLUSIONES}

Los ejemplos de la tercera Centuria analizados constatan la importancia de la alimentación en el pro- 
ceso curativo de los pacientes tratados por Amato Lusitano. Ya sea en forma de meras referencias (optimo victus ordine constituto; cum debito victus ordine describo) o en prolijas descripciones a modo de regimina, observamos la preocupación del portugués por ofrecer al enfermo una dieta individualizada, adaptada a su naturaleza y temperamento y específica para cada enfermedad. Sus consejos dietéticos, muy ligados a la terapia, constituyen una importante vía de curación $y$, como tales, deben ser respetados por el paciente como paso previo a su recuperación.

El conjunto de alimentos $-y$ solo hemos recogido una parte- parece el común en su tiempo. Hallamos una despensa amplia en la que son más frecuentes las aves, mucho más que la carne de animales de mayor tamaño, y donde la porcina es muy a menudo omitida. Desconocemos si esta decisión obedece a sus convicciones religiosas, pues, aunque nuestro autor nace ya en una familia de cristianos nuevos, está comprobado que los hábitos alimentarios tardan en cambiarse al menos dos generaciones (Motis Dolader, 1995, p. 210) ${ }^{27}$.

No hay una división clara entre cocina y dietética, pues son varias las ocasiones en las que se incluyen recetas e instrucciones sobre el modo de preparación y conservación de los platos, las mismas que en los libros de cocina de la época. A esto se suma el hecho

\section{NOTAS}

1 Este trabajo se inscribe en el seno del Proyecto de Investigación "Estudios de medicina práctica en el Renacimiento: Las Centurias de Amato Lusitano II", subvencionado por la Agencia Estatal de Investigación del Gobierno de España (ref. FFI2017-82381-P) y por la Junta de Castilla y León (ref. VA222P20).

2 Una amplia bibliografía sobre este autor puede verse en la página del mencionado proyecto. http://amatolusitano.uva. es/bibliografia/ [consultado el 30/01/2020]. Sobre su cronología cf. Blanco Pérez, 2019.

3 La publicación de las Centurias de Amato es compleja. Hasta obtener la edición crítica de las mismas, citamos la edición de Burdeos 1620. En las referencias a la obra señalamos el número de la Centuria (.) y de la curatio, en ocasiones seguida del título. Las traducciones son nuestras.

4 Los Regimina Sanitatis medievales recomiendan una dieta tenuis o subtilis, basada en alimentos delicados como el poIlo, el capón o la ternera, a personas de vida poco activa. En cambio, se recomienda una dieta gruesa para campesinos o atletas que necesitan reponerse tras un gran esfuerzo (Gil Sotres, 1996, pp. 649-650). de que muchos alimentos se prescriben también como medicamentos.

Estamos ante una despensa de lujo, pues el portugués se sirve sobre todo de lo que él mismo denomina cibi optimi et selecti, productos de prestigio y muy costosos entonces. Los alimentos óptimos tenían por sí mismos un valor externo, pues la relación entre alimentación y jerarquía social estaba claramente asentada (Grieco, 2004). Además, hemos podido comprobar que a pacientes con menos recursos les prescribe otros productos más simples y toscos.

En definitiva, optimo victus ordine constituto se revela como un importante principio de actuación para Amato. Vemos que está omnipresente en su práctica o experientia y que cuando reflexiona sobre ello, como buen médico de formación superior, no puede desligarse de la referencia a las autoridades que dominan la enseñanza universitaria. Bien es cierto que tiende a interpretar a estos autores de la forma más acorde a su pensamiento e intereses.

Es este un procedimiento terapéutico que además de situarnos en la órbita de la medicina de su tiempo nos permite acercarnos a otros aspectos sociales o antropológicos, pues en el fondo recoge una fotografía de cierta cocina y ciertos hábitos alimentarios en el siglo XVI.

5 Por el principio terapéutico contraria contrariis curantur remedios fríos y secos convienen a enfermedades cálidas y húmedas; y remedios secantes a afecciones húmedas.

6 En 3.71, de febre continua maligna interficiente, ofrece la relación de los alimentos que confortan y revigorizan (ad ea quae roborant et vires reficiunt confugimus).

7 Existía un gran debate sobre si la cena debía ser más abundante que la comida. Cristóbal de Vega en De arte medendi (Vellisca Gutiérrez, 2018, pp. 255-256) comenta que esta era una duda habitual entre los iuniores medici. La mayoría de los autores prefieren la cena como comida principal, pues así se realiza la digestión durante el sueño reparador (Gil Sotres, 1996, pp. 655-666). No obstante, el propio Amato en otras curas recomienda una cena más abundante $(3.87 ; 3.91)$.

8 Sobre el uso del vino en Amato cf. González Manjarrés, 2017.

9 En general, se desaconseja el sueño diurno cf. Avicena, 1527, 1, 3, 2, 9,50rb.

103.1 , semel vero hectica laborantes magna (sic) ciborum ingerere copiam malum est; nam iis omne nimium pessimum est, praecipue quod vires resolvere potest, ut immodicus cibus, frequens motus, magnum exercitium et similia. "Es 
perjudicial que los que sufren de fiebre héctica ingieran una gran cantidad de alimentos, principalmente porque el exceso de comida, un ejercicio frecuente, una gran actividad física y similares pueden consumirles las fuerzas".

11 El francolín fue un manjar en las mesas europeas del siglo XVI. Según Cristóbal de Vega (Vellisca Gutiérrez, 2018, p. 214) fue traído de Nápoles al reino de Valencia en tiempos de Fernando el Católico. Como bocado exquisito lo menciona Cervantes en El Quijote (II, cap. 49).

12 3.87, gallinas Indicas, Africanas dictas, quas Graecia meleagrides appellare solet, quarum carnes phasianis anteferuntur; ita enim delicatae et friabiles hodie Romae habentur ut omnium avium carnes facile superare dicantur. "Gallinas de Indias, o africanas, que en Grecia suelen llamarse meleagrides (Numida Meleagris L.), cuya carne se prefiere a la de los faisanes; en Roma se consideran hoy tan delicadas y tiernas que se dice que superan fácilmente a la carne de las demás aves".

13 Para este autor remitimos a la edición de Hipócrates (18391861) que abreviamos como $L$.

14 Se trata de un plato común en la época, que aparece también en libros de cocina como plato especial para enfermos. Por ejemplo, Francisco Martínez Motiño, cocinero de Felipe III y Felipe IV, en Arte de cozina, pastelería, vizcochería y conservería (1611) incluye una receta de "pistos para enfermos" (ff. 246r-246v) o Bartolomeo Scappi (1570), cocinero, entre otros, del Papa Julio III, a quien atendió Amato durante su estancia en Roma (2.31), en el libro sexto, dedicado a convalescenti, recoge recetas de pisto de capón, pollo y ternera (cap. 30-35) (ff. 397v-399r).

15 La ptisana es una especie de caldo espeso o papilla a base de cebada. Cremor ptisanae, expresión ya atestiguada en Celso $(4,13,5)$, designa el residuo que se obtiene tras colarla y que los árabes denominaban sauich hordei: 3.15: ptisanae cremor, quem colatam ptisanam appellamus, Arabes sauich hordei appellant. (Cf. Vázquez de Benito - Herrera Hernández, 1989, pp. 196-197).

16 DRAE (s.v.) "1. Especie de papas que se hacen con caldo muy sustancioso y pan rallado, a lo cual se suele agregar gallina picada, yemas de huevo, azúcar u otros ingredientes". De nuevo los libros de cocina lo registran como preparaciones destinadas a enfermos. Cf. Martínez Motiño, 1611, ff. $247 r-248 r$ y Scappi, 1570, f. 407r.

17 Encontramos también en el libro sexto de Scappi, a quien se atribuye un origen veneciano $o$, al menos de la zona norte, dos recetas de unos morselleti o mostaccioli (cap. 142-143) que contienen uno o dos granos de almizcle (Scappi, 1570, ff. 420r-420v).

18 Hallamos ambos términos en los libros de cocina. En Bartolomeo Scappi leemos acqua con orzo "agua de cebada") (1570, f. 392v) y la orzata d’orzo (ff. 403v-404v), bebida más contundente obtenida tras cocer cebada y otros elementos como arroz, almendras, pepitas de melón o chufas acompañada incluso de caldo de capón.
19 Camillo Colonna, como es tradición en su familia, es defensor de la facción imperial frente al Papa. Desde 1525 participa en diversos acontecimientos como el sitio de Florencia (1529) a las órdenes del príncipe de Orange o en el asedio de Siena (1552) a las de García de Toledo. Apoya a Cosme de Medici cuando inició una guerra contra esta ciudad. También intervino en varias batallas a favor del emperador Carlos V contra los turcos (Argel, Düren). En 1555 fue acusado de conjurar contra el papa Pablo IV y tras ser liberado muere en Roma en 1558 (Petrucci, 1982).

20 La mayoría de las curaciones que integran la Curationum Centuria Tertia son de pacientes tratados en Ancona, excepto un pequeño grupo que responde a casos atendidos en Roma (Andretta, 2019). Allí acude favorecido por la familia del gobernador de Ancona para atender al Papa Julio III, afectado de morbo gallico, aunque en la curación específicamente dedicada a él (2.31) jamás se mencione que padeciera tal enfermedad (Pérez lbáñez, 2019).

21 Poco después durante el asedio de Siena en 1552 le sobrevino un ataque de gota (Petrucci, 1982), por lo que suponemos que ya empezara a manifestar síntomas de ella.

22 Para las referencias a este autor remitimos a la edición de Galeno (1821-1833), que abreviamos como K.

23 Así se titula en la traducción de Niccoló da Reggio y en la versión de Leonardo lacchino reproducida en Galeno (1541-1545, vol. 3). Con el título De praenotione ad Epigenem aparece en K. La cita de Amato es puramente ideológica basada, entendemos, en las conclusiones que pueden inferirse del caso de la mujer enamorada del bailarín y del ecónomo agobiado por una posible pérdida monetaria (6 [K. 14.630-635]).

24 Tópico recurrente en sus Centurias (Vellisca Gutiérrez, 2019).

25 En el Introitus Amato ya había establecido que el enfermo debe obedecer al médico (Aeger quoque [...] obediens medico sit oportet). Más aún, el paciente debe comportarse como un esclavo con su amo (ipse aeger parere medico tanquam servus domino debet) hasta el punto de que prohíbe dar consejo médico a aquellos que no sigan sus instrucciones (iis qui praeceptis non obsequuntur, medicinale consilium dandum non est), frase que repite literalmente en estos scholia.

26 Conocedor del carácter afrodisiaco atribuido a bivalvos y crustáceos, limita su prescripción por impuros (Levítico 11,9-10).

27 Amato como otras familias conversas, son seres "liminales", en palabras de David M. Gitlitz (2010, p. 90), que intentan disimular su herencia judía, pero en muchos momentos de la vida cotidiana les costaba renunciar a ella, especialmente en los hábitos alimentarios. 


\section{BIBLIOGRAFÍA}

Albala, Ken (2002), Eating Right in the Renaissance, Berkeley - Los Angeles - London, University of California Press.

Álvarez de Palacio, Eduardo (2008), “El esquema galénico de las sex res non naturales como fundamento del concepto de salud corporal en el Humanismo renacentista español". En: Nieto Ibáñez, Jesús María; Manchón Gómez, Raúl (eds.), El humanismo español entre el viejo mundo y el nuevo, León, Universidad de León, Secretariado de Publicaciones; Jaén, Universidad de Jaén, Servicio de Publicaciones, pp. 255-274.

Amato Lusitano (1620), Curationum Medicinalium Centuriae septem, Burdigalae, Gilbert Vernoy.

Andretta, Elisa (2011), Roma medica. Anatomie d'un systéme medical au XVI siècle, Roma, École française de Rome.

Andretta, Elisa (2019), "La Roma di Amato Lusitano (1550-1551). Attori, luoghi, pratiche professionali e intelletuale". En: González Manjarrés, Miguel Ángel (ed.), Praxi theoremata coniungamus. Amato Lusitano y la medicina de su tiempo, Madrid, Guillermo Escolar, pp. 81-99.

Avicena (1527), Liber Canonis medicine cum castigationibus Andreae Bellunensis, in edibus Luce Antonii lunta Florentini, Venetiis.

Banegas López, Ramón A. (2012), Europa carnívora: comprar y comer carne en el mundo urbano bajomedieval, Gijón, Trea.

Blanco Pérez, José Ignacio (2019), “Cronología de Amato Lusitano. Amato Lusitano y su época”. En: González Manjarrés, Miguel Ángel (ed.), Praxi theoremata coniungamus. Amato Lusitano y la medicina de su tiempo, Madrid, Guillermo Escolar, pp. 287306.

Cavallo, Sandra; Storey, Tessa (2013), Healthy living in Late Renaissance Italy, Oxford, Oxford University Press.

Celso (1915), A. Cornelii Celsi quae supersunt, De medicina. Marx, Friedrich (ed.), Leipzig-Berlin, Teubner.

Galeno (1541-1545), Operum omnium sectiones prima-octava, Ricchi, Agostino (ed.), Venetiis, Farri.

Galeno (1821-1833), Claudii Galeni opera omnia, Kühn, Karl G. (ed.), 20 vol., Leipzig, Cnobloch (repr. Hildesheim, Olms, 19641965).

Gil Sotres, Pedro (1996), "Introducción”. En: García Ballester, Luis; Paniagua, José Antonio; McVaugh, Michael R. (eds.), Arnaldi de Villanova Opera Medica Omnia X.1. Regimen Santitis ad regem Aragonum, Barcelona, Edicions Universitat Barcelona, pp. 471-885.

Gitlitz, David M. (2010), "Conversos, ollas e inquisidores: duelos y quebrantos". En: Izquierdo Benito, Ricardo; Macías, Uriel (eds.), La mesa puesta: leyes, costumbres y recetas judías, Cuenca, Ediciones de la Universidad de Castilla-La Mancha, pp. 89-106.

González Manjarrés, Miguel Ángel (2017), “En el taller de Amato: un escolio sobre la fiebre y el vino", Euphrosyne, 45, pp. 341-352.
Grieco, Allen F. (2004), "Alimentación y clases sociales a finales de la Edad Media y en el Renacimiento". En: Flandrin, JeanLouis; Montanari, Massimo (eds.), Historia de la alimentación, Gijón, Ediciones Trea, pp. 611-624.

Herrera Hernández, María Teresa (1996), Diccionario español de textos médicos antiguos, Madrid, Arco Libros.

Hipócrates (1839-1861), Oeuvres complètes d'Hippocrate, Littré, Émile (ed.), 10 vol., Paris, J.B. Baillière (reimpr. Amsterdam 1978).

Martínez Motiño, Francisco (1611), Arte de cozina, pasteleria, vizcocheria y conserveria, Madrid, Luis Sánchez.

Motis Dolader, Miguel Ángel (1995), "Régimen alimentario de las comunidades judías y conversas en la Corona de Aragón en la Edad Media". En: Actes: 1r. Col.loqui d'Història de l'Alimentació a la Corona d’Aragó. Edat Mitjana, Vol. 1., Lleida, Instituto de Estudios Ilerdenses, pp. 205-362.

Nicoud, Marilyn (2007), Les Régimes de santé au Moyen Âge: Naissance et diffusion d'une écriture médicale (XIIIe-XV_siècle), Rome, École française de Rome.

Pérez Ibáñez, María Jesús (2019), “Amato ante el morbo Gálico”. En: González Manjarrés, Miguel Ángel (ed.), Praxi theoremata coniungamus. Amato Lusitano y la medicina de su tiempo, Madrid, Guillermo Escolar, pp. 179-200.

Petrucci, Franca (1982), “Colonna, Camillo”. En: Dizionario Biografico degli Italiani, 27. http://www.treccani.it/ enciclopedia/camillo-colonna_res-0530f0cc-87eb-11dc8e9d-0016357eee51_\%28Dizionario-Biografico\%29/ [consultado el 24/02/2020].

Pomata, Gianna (2010), "Sharing cases: The Observationes in Early Modern Medicine", Early Science and Medicine, 15, pp. 193-236.

Recio Muñoz, Victoria (2018), "Ut iuvet et non noceat: médico, paciente y enfermedad en el Introitus de Amato Lusitano a las Curationum Medicinalium Centuriae", Euphrosyne, 46, pp. 261-277.

Scappi, Bartolomeo (1570), Opera di M. Bartolomeo Scappi, cuoco secreto di papa Pio V, Venetia, Tramezzino.

Vázquez de Benito, María Concepción; Herrera Hernández, María Teresa (1989), Los arabismos de los textos médicos latinos y castellanos de la Edad Media y la Modernidad, Madrid, Consejo Superior de Investigaciones Científicas.

Vellisca Gutiérrez, Nelia (2018), Cristóbal De Vega. Sobre el arte de curar. Traducción anotada del libro // De arte medendi. El cuidado de la salud en la España del siglo XVI, Valladolid, Ediciones Universidad de Valladolid.

Vellisca Gutiérrez, Nelia (2019), “Diálogo de un médico con la avaricia (Amato, Centurias, 2,53)", eHumanista/Conversos, 7, pp. 59-75. 